\title{
THE ONE TO MULTIPLE AUTOMATIC HIGH ACCURACY REGISTRATION OF TERRESTRIAL LIDAR AND OPTICAL IMAGES
}

\author{
Wang $\mathrm{Yu}^{123}$, Hu Chunmei ${ }^{123}$, Xia Guofang $^{123}$, Xue Huimin ${ }^{123}$ \\ ${ }^{1}$ School of Geomantic and Urban Information, Beijing University of Civil Engineering and Architecture, NO.15 Yongyuan Road, \\ Daxing District, Beijing, 102616 - (Hu Chunmei, Wang Yu, Xia Guofang, Xue Huimin)@bucea.edu.cn \\ ${ }^{2}$ Beijing, Key Laboratory for fine reconstruction and health monitoring of Architectural Heritage, NO.15Yongyuan Road, Daxing \\ District, Beijing, 102616 \\ ${ }^{3}$ Engineering Research Center of Representative Building and Architectural Heritage Database,Ministry of Education,Beijing , \\ 100044,China
}

Commission III, WG III/5

KEY WORDS: Ground Lidar, Optical Image, Automatic, High Precision, Registration

\begin{abstract}
:
The registration of ground laser point cloud and close-range image is the key content of high-precision 3D reconstruction of cultural relic object. In view of the requirement of high texture resolution in the field of cultural relic at present, The registration of point cloud and image data in object reconstruction will result in the problem of point cloud to multiple images. In the current commercial software , the two pairs of registration of the two kinds of data are realized by manually dividing point cloud data , manual matching point cloud and image data , manually selecting a two - dimensional point of the same name of the image and the point cloud , and the process not only greatly reduces the working efficiency, but also affects the precision of the registration of the two , and causes the problem of the color point cloud texture joint. In order to solve the above problems, this paper takes the whole object image as the intermediate data, and uses the matching technology to realize the automatic one-to-one correspondence between the point cloud and multiple images. The matching of point cloud center projection reflection intensity image and optical image is applied to realize the automatic matching of the same name feature points, and the Rodrigo matrix spatial similarity transformation model and weight selection iteration are used to realize the automatic registration of the two kinds of data with high accuracy. This method is expected to serve for the high precision and high efficiency automatic 3D reconstruction of cultural relic objects, which has certain scientific research value and practical significance
\end{abstract}

\section{INTRODUCTION}

The automatic registration of ground 3D laser point cloud and close-range images is a difficult problem in the registration of two kinds of non-homologous data. At present, the automatic registration of the above two source data is realized mainly through the matching of the ground three-dimensional laser reflection intensity image and the close range image. Automatic registration is divided into two parts: the automatic correspondence of the high resolution image and the point cloud, and the matching and registration of the same name points of the point cloud and the image.

Now, there has been a lot of research progress in automatic registration methods, In the matching of basic elements, it is mainly reflected in the feature points of the same name, the automatic extraction of the line of the same name points, most of the research focuses on airborne images and airborne radar data with POS data; The registration methods include collinear equation method, angular cone method, direct linear transformation method, and direct solution based on Rodrigo matrix. There is no auxiliary information such as POS in the laser radar data of the objects of cultural relics on the ground, Its image data does not have the topological information that aerial images have, or the one-to-one correspondence between the image and the corresponding point cloud, the extraction of the two data homonyms brings some difficulty.

The large amount of point cloud data and the large number of high resolution images are the main factors that affect the automation. In response to the above problems, in this paper, it is proposed that the projective reflection intensity image and the corresponding global image can be used as mediators. Using image matching and other techniques to realize the high resolution image and point cloud one to one correspondence, after extracting the same name feature automatically, the Rodrigo matrix mathematical model and the iterative method of weight selection are used to obtain the high precision registration parameters. The specific methods are provided as follows:

1. Generation of Point Cloud reflection intensity Center projection Image and RGB Image: Firstly, the object point cloud data are acquired by the ground laser scanner, and the maximum outlier box of the point cloud is calculated.; Then, the image datum level of point cloud reflection intensity image is determined by the wrapped box, the size of reflection image is determined by the intersection region of laser ray and datum plane, and the grid of reflected image is determined by point cloud resolution; Finally, the projection reflectance intensity image and RGB image of

\footnotetext{
* Corresponding author: huchunmei@buca.edu.cn
} 
point cloud center are generated by nearest neighbor interpolation.

2. One to multiple matching of point cloud and images: According to each point cloud partition, the whole image of the region is obtained, and the local high resolution image with the required resolution is obtained. Firstly, the point cloud RGB image and the whole image are matched to make the whole image correspond to the point cloud one by one. According to the matching of the whole image and the local image, the location of the local image in the whole image is determined. According to the above results, the local image and the point cloud are automatically oneto-one corresponding with the whole image as the intermediary.

3. Automatic high accuracy registration of point cloud and images: For the point cloud data corresponding to the local high precision image, the center projection reflection intensity image and the RGB image are generated according to the method of 1 , and the point cloud RGB image is used to match the corresponding optical image with the point cloud RGB image. The rough registration of two kinds of data at any angle is realized by using the feature points of the same name and the registration model based on Rodrigo matrix. In high precision iterative least square registration collinearity equation, standardized residuals Danish method using improved iteration method, the crude almost deweight, with iteration, outliers weight of observation value will become more and more small, to zero, the iteration terminates, and the adjustment results will no longer be the influence of gross error. The collinear equation is used as the mathematical model and the texture mapping is used to generate the seamless color point cloud model.

\section{METHOD}

For an object, to take block photography in a certain way. For example, buildings can be built in ways such as east, west, north, south, inside and outside, the corresponding point cloud data are also partitioned in the same way. In order to match the global optical images of each object, the focal point cloud is used to generate the center plane projection reflection intensity image and the RGB color image, and the relation between the block point cloud and its optical image is established.

\section{1 point cloud center plane projection image generatio}

Central projection is the projection mode in which projection rays converge at a point. The main ideas and steps are as follows:

Determine the point cloud center point projection plane. The center point of 3D laser scanner is the projection center and the center point of 3D laser point cloud is the Projection center point. A projection plane is a plane of lines perpendicular to the point at the center of the projection to the center of the cloud. Here is the projection plane formula:

$$
\left(\mathrm{x}_{\mathrm{c}}-\mathrm{x}_{0}\right)\left(\mathrm{x}-\mathrm{x}_{\mathrm{c}}\right)+\left(\mathrm{y}_{\mathrm{c}}-\mathrm{y}_{0}\right)\left(\mathrm{y}-\mathrm{y}_{\mathrm{c}}\right)+\left(\mathrm{z}_{\mathrm{c}}-\mathrm{z}_{0}\right)\left(\mathrm{z}-\mathrm{z}_{\mathrm{c}}\right)=0
$$

And then the point where the projection ray intersects the plane from each point in the projection center toward the point cloud is calculated. That is, the projection point of a point in the point cloud on the projection plane.As shown in figure 1.

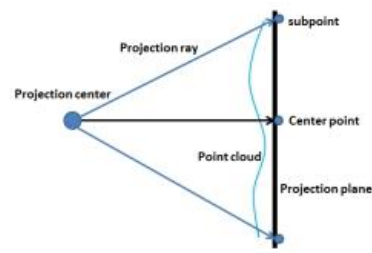

Figure 1.The point cloud is projected to the plane by the central projection

Secondly, the right angle coordinate system of projection plane space is established. Calculate the minimum outer rectangle of all projection points in the projection plane, take the upper-left corner of the rectangle as the origin of the axis $\mathrm{O}$, the eastward direction of the minimum outer rectangular edge from the origin is an axis $\mathrm{X}$, to the south is the axis $\mathrm{Y}$, vertical plane $\mathrm{XOY}$ as axis Y. Establishment of Spatial Cartesian coordinate system of projection plane O-XYZ.As shown in figure 2.The spatial linear equations of the $\mathrm{OX}$ axis and the $\mathrm{OY}$ axis are obtained under the coordinate system of the $3 \mathrm{D}$ laser scanner.

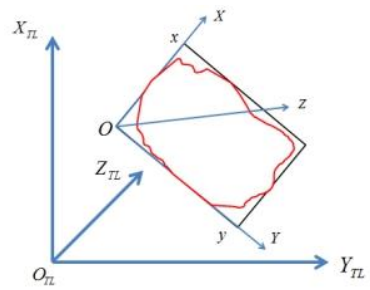

Figure 2. Projection plane coordinate diagram

Finally, the projection plane coordinate system is established. Because all points are in the XOY plane, the $\mathrm{Z}$ value of the projection point in the $\mathrm{O}-\mathrm{XYZ}$ coordinate system is all 0 . The distance between each projection point on the projection plane and the $\mathrm{O}-\mathrm{XYZ}$ axis of the projection plane space right-angle coordinate system $\mathrm{O}-\mathrm{XYZ}$ is calculated. The distance is regarded as the coordinate of the projection point in the XOY plane Cartesian coordinate system. Starting from the origin of the XOY plane in the spatial Cartesian coordinate system of the projection plane, the sampling interval of the point cloud is taken as the step size. Row by row search for the nearest point in the projection plane to the nearest lattice dot in a domain of point cloud sampling intervals, using the laser reflection intensity of the nearest point as the Is the gray value of the grid dot and generates a laser reflection intensity image. Figure 3 .

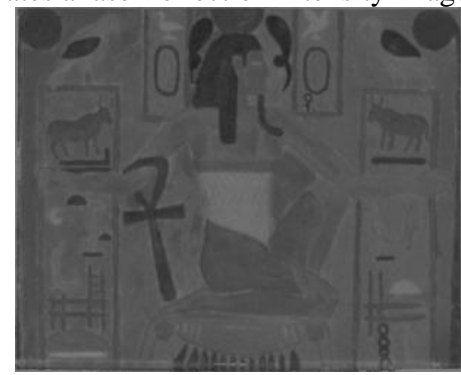

Figure 3. Laser reflection intensity 


\subsection{One-to-one correspondence between local images and heir corresponding point clouds}

According to the reflection intensity image and RGB image of the projecting point cloud in the center of the object block, the whole image of the object and the local image data with high resolution are obtained by using the digital camera. The pixel resolution of the whole image is adjusted so that the resolution of the whole image is basically the same as that of the point cloud RGB image. The corresponding relationship between the point cloud of each block and the whole image is established by using SIFT matching to obtain the point of the same name. The high resolution local image data and the whole image are matched by SIFT and the corresponding transformation relationship is established.

The one-to-one correspondence between the local image and the global image is established, and the affine variation of the local image and the global image is established according to the matching point of the same name. The relationship between the local high resolution image and the point cloud reflection intensity image can be obtained by using the whole image as the intermediary. According to the affine transformation parameters the minimum outer rectangle of the point cloud reflection intensity image can be obtained. According to the 3D coordinates of the point cloud reflection intensity image in the region, the minimum outsourced box algorithm is used to calculate the point cloud region corresponding to the highresolution image. And the point cloud and image of the high accuracy registration, the basic process is shown in figure 4 .

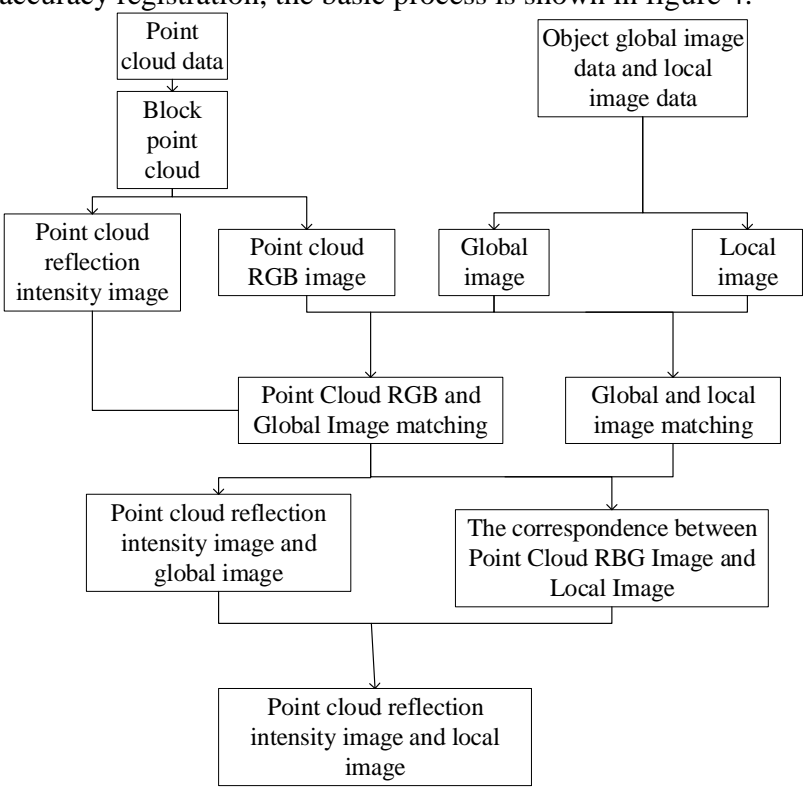

Figure 4. One-to-one flow chart of point cloud and image

2.2.1 matching between Point Cloud RGB Image and Global Image: In this paper, the scale invariant feature transform (SIFT) algorithm is used to match the reflected intensity image with the global image. The main idea of SIFT algorithm is to transform the matching between images into the similarity measure between feature point vectors .

Firstly, the stable features are extracted and described in the scale space, and then the generated feature vectors are matched. The main steps of SIFT matching include five steps: the extreme detection of scale space, the precise location of key points, the determination of the main direction of key points, the description of key points and the matching of key points. The detection of the extreme value of the scale space is mainly through the scale transformation of the original point cloud to obtain the scale space representation sequences under the multiscale image. The main contour of these sequences is extracted in the scale space, and the main contour is used as a feature vector. Edge detection, corner detection at different resolution of the key point extraction and so on. The construction of scale space is based on the DOG pyramid. In order to find the extremum of the DOG function, each pixel should be compared with all its adjacent points to see if it is larger or smaller than its adjacent points in the image domain and the scale space domain. The accurate location of the key points. DOG is sensitive to noise and edges, so the local extremum detected in the scale space of the second step has to be further screened to remove the unstable and error-detected extreme points.

In this way, the performance of matching can be enhanced, the result of matching is more stable and the ability of resisting noise is strong. The main direction of the key points is determined. The stable extreme points are extracted under different scale spaces, which ensures the scale invariance of the key points . The problem to be solved for the key distribution direction information is that the key points have the invariance to the image angle and the rotation . The direction assignment is realized by finding the gradient of each extreme point . Key point description. The description of key points is a key step in the subsequent implementation of matching. In fact, the description is a kind of mathematical definition of the key process. The descriptor includes not only the key points, but also the neighborhood points around the key points.

Key points match. The matching of feature points is realized by calculating the Euclidean distance of 128 dimensional key points of two groups of feature points. The smaller the Euclidean distance, the higher the similarity. When the Euclidean distance is less than the set threshold, it can be judged as a successful match as shown in figure 5 .

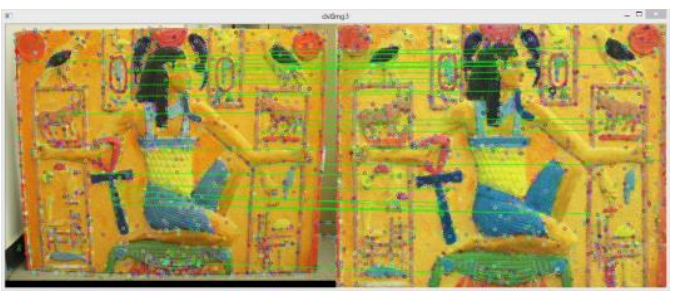

Figure 5. Matching results of optical images with point cloud RGB images

2.2.2 Matching of global image and local image: For the local high resolution image corresponding to the global image, the transformation relationship between the images needs to be solved through the SIFT algorithm to calculate the matching feature. Affine transformation can well express the general transformation between images, and needs at least 3 pairs of matching points to solve. This paper will use the RANSAC method to estimate the affine transformation relationship between the two images. If there are enough image features matching to conform to the particular affine transformation, we can argue that the current matching is considered to be 
satisfactory, whereas the next estimation is carried out until a sufficient number of features are found to satisfy affine transformation, and the affine transformation parameter model is obtained as formulae (2):

$$
X_{l}=\gamma X+t
$$

where $\mathrm{X}$ represents the coordinate vector before and after transformation

$\lambda=$ rotation matrix

$\mathrm{t}=$ translation matrix

The matching results are shown in figure 6 .

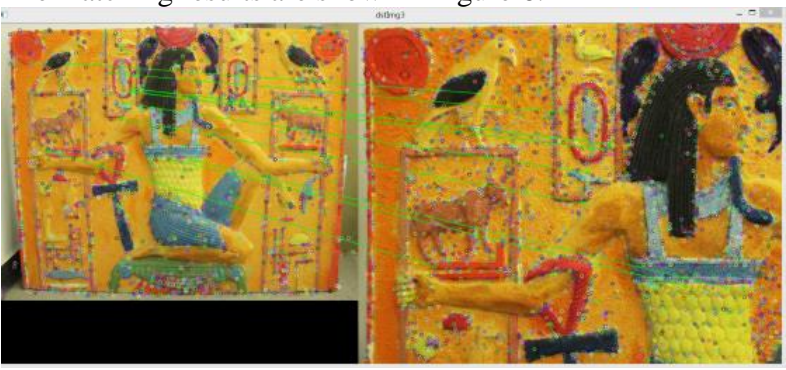

Figure 6. Local image matching

2.2.3 The local image corresponds to the one by one: Through the global image as the medium, the high-resolution local image can be solved by affine transformation parameter model, and it can find the local RGB point cloud image and reflection intensity image corresponding to the point cloud RGB image, and generate the local point cloud RGB image and reflection intensity image by using the minimum outlier rectangle algorithm.

According to the minimum outsourcing box of the minimum outsourcing rectangle of the reflection intensity image, the point cloud data in the minimum outsourcing box is searched and the local point cloud is segmented. At this point, the local point cloud corresponds to the high resolution image, as shown in figure 7 .

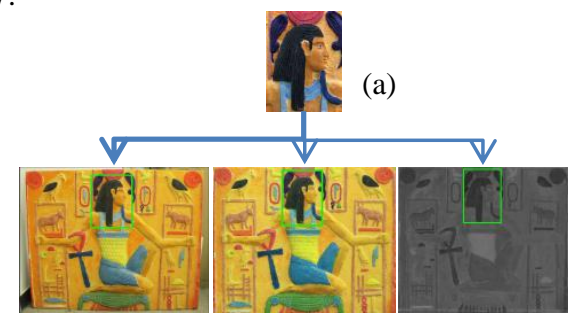

(b)

(c)

(d)

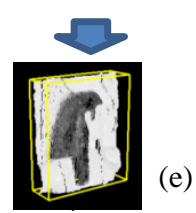

Figure 7. Point clouds corresponding to local images

a) Local image, b) Global image, c) RGB point cloud image, d) Ppoint cloud gray image, e) Bureau local image corresponding point cloud region
2.2.4 Acquire point cloud and homonymy points in images: Through the SIFT matching between the local image and the local point cloud RGB image, the RANSAC method and the bidirectional consistency matching method are used to eliminate the errors, and the correct matching points with the same name are obtained. The local point cloud RGB image is used as the intermediary, and the matching result between the reflection intensity image and the local optical image is consistent with that of the point cloud RGB image and the local optical image. As shown in figure 8 , it is the point cloud RGB image matching with the optical image.

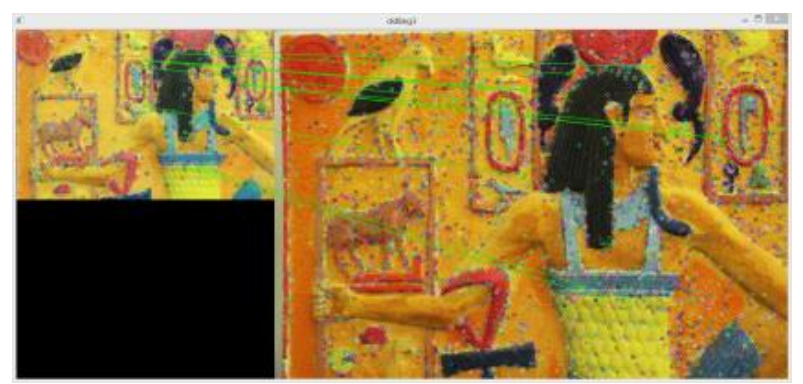

Figure 8. Acquisition of points with the same name from local images and point cloud RGB images

2.2.5 High Precision automatic Registration of Point Cloud and Image: The registration of laser point cloud and image is a three-dimensional and two-dimensional registration problem, which mainly solves two problems: One is the registration of the basic element, the other is the registration method.

For the allocation of basic elements, mainly a bit, line two ways. Because of the difference in resolution between laser point cloud and texture image, the acquisition of collocation elements is mainly manual without special marks. At the same time, because of the uncertainty of the set of scanned objects and the uncertainty of shooting angle, the extraction of line features affected by texture image and point cloud also has great limitations.

The registration methods include collinear equation method, angular cone method, direct linear transformation method, and direct solution based on Rodrigo matrix. Through the analysis, the collinear equation solution and the angular cone solution need better initial value, and complete the calculation by iteration, the direct linear transformation method has certain requirements to the control point, when the control point distribution or approximate distribution is in one plane, the result is wrong. And need six pairs of more than the same name points; The direct solution to the Rodrigo matrix is mainly through three pairs of control points. The transformation model is not strict enough to calculate the external elements of the image by the addition, subtraction and multiplication of the matrix. In this paper, a registration method from coarse to fine is used. The matching feature point obtained from section 2.2.4 is the matching primitive element. Firstly, the barycenter coordinate is used to simplify the spatial similarity transformation model formula (3) 


$$
\left[\begin{array}{c}
X_{m} \\
Y_{m} \\
Z_{m}
\end{array}\right]=\lambda R\left[\begin{array}{c}
x_{m} \\
y_{m} \\
0
\end{array}\right]
$$

where $\left[X_{m}, Y_{m}, Z_{m}\right],\left[x_{m}, y_{m} 0\right]=$ centroid coordinates

$\lambda=$ scaling coefficient

$\mathrm{R}=$ rotation matrix

Combining the properties of antisymmetric matrix formula (4) and orthogonal matrix formula (5), the model formula of registration parameter angular element is obtained (6).

$$
\begin{gathered}
\mathrm{S}=\left[\begin{array}{ccc}
0 & -c & -b \\
c & 0 & -a \\
b & a & 0
\end{array}\right] \\
R=(I+S)(I-S)^{-1} \\
{\left[\begin{array}{ccc}
0 & -Z_{m} & -Y_{m}-\lambda y_{m} \\
-Z_{m} & 0 & X_{m}+\lambda x_{m} \\
Y_{m}+\lambda y_{m} & X_{m}+\lambda x_{m} & 0
\end{array}\right]\left[\begin{array}{l}
a \\
b \\
c
\end{array}\right]-\left[\begin{array}{l}
X_{m}-x_{m} \\
Y_{m}-y_{m} \\
Z_{m}-z_{m}
\end{array}\right]=0}
\end{gathered}
$$

Then the improved iterative method of weight selection of the standardized residual Denmark method is used to reduce the weight of the small gross error until the registration meets the precision requirements.

\section{EXPERIMENT AND ANALYSIS}

\subsection{Experimental data}

In this paper, a single side relief of ancient Egyptian sandstone slate is taken as an example.The experimental material is $1 \mathrm{~m} \mathrm{~L}$, 0. $8 \mathrm{~m} \mathrm{~W}, 0.25 \mathrm{~m}$ thick. FARO T330 is used to obtain 3D Terrestrisl Laser data, and Cannon 600D is used to obtain close range image. The sampling interval of point cloud is $2 \mathrm{~mm}$, total 157528 scanning points. The advantages and reliability of this method are verified by experimental data.

\subsection{Analysis}

Combined with the above experimental data, the method is realized by using VS2013 platform and OpenCV3.1 PCL point cloud library. The near view image data are obtained from the point cloud data, and the close range image data includes the whole image and the local high resolution image.

1. By establishing the center projection plane and taking the resolution of the point cloud as the step size, the grid is established, and the reflection intensity value of the near point is assigned to the grid dot by searching the adjacent point, and the reflection intensity image is generated. In the same way, the RGB value of the adjacent point is assigned to the lattice dot to generate the point cloud RGB image, as shown in figure 9 and figure 10 ;

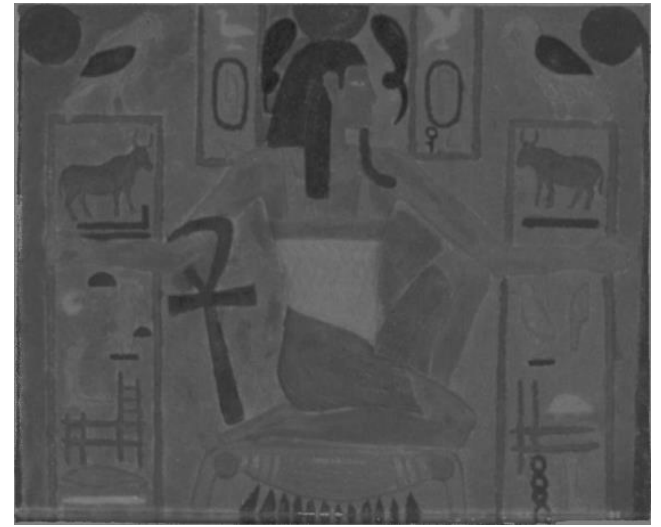

Figure 9. Point Cloud reflection intensity Image

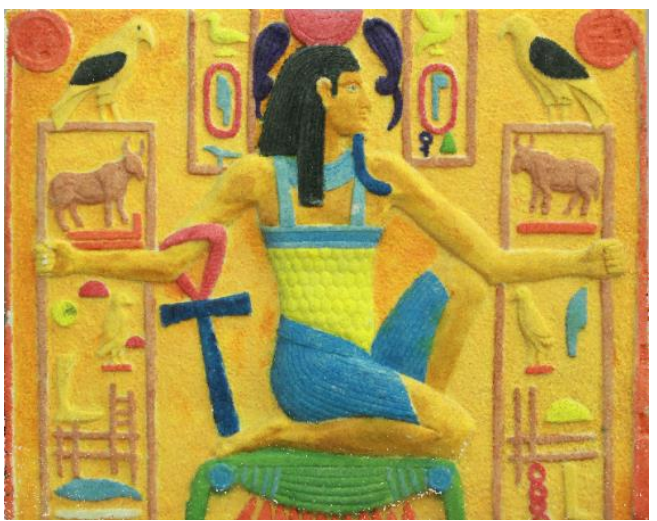

Figure 10.Point Cloud RGB Image

2. The result of SIFT matching between global image and point cloud RGB image is shown in figure 11;

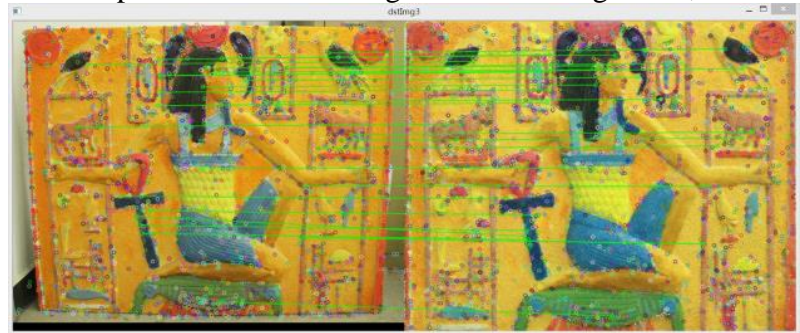

Figure 11. Registration results of global image and point cloud image

3. The local image is matched with the whole image, and the local image corresponding to the local point cloud RGB image is obtained by parameter conversion, such as figure 12 , figure 13 , figure 14 ;

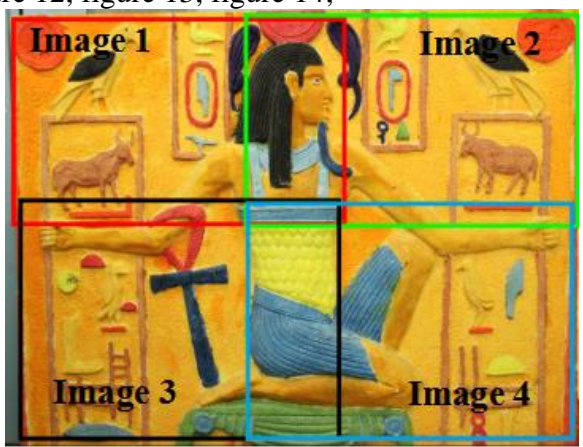

Figure 12. One to one correspondence between local image and whole image 


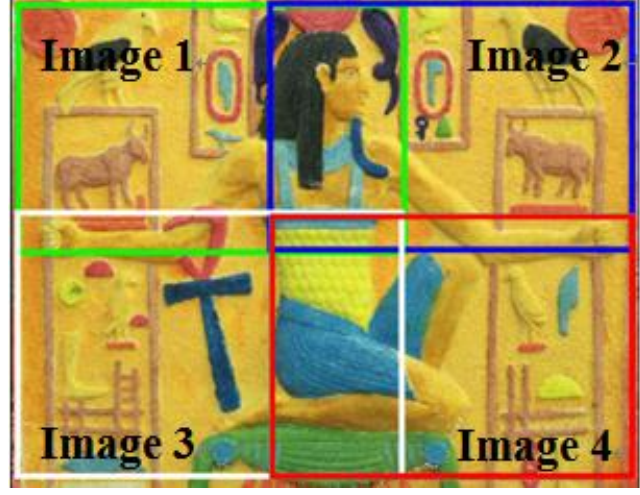

Figure 13.One to one correspondence between local image and point cloud RGB image

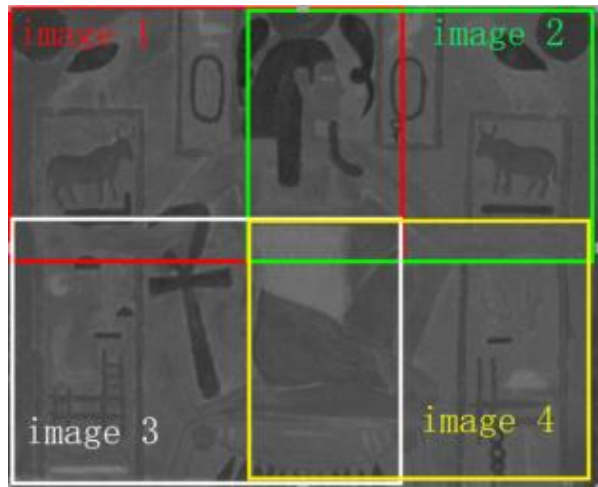

Figure 14. One to one correspondence between local image and point cloud

4. The feature points of the same name can be obtained by high precision matching between local RGB images and local images. Since the point cloud reflection intensity image is generated by the same method as the point cloud RGB image, the same feature points of the same name can also be obtained on the point cloud reflection intensity image, as shown in figure 15 and 16 ;

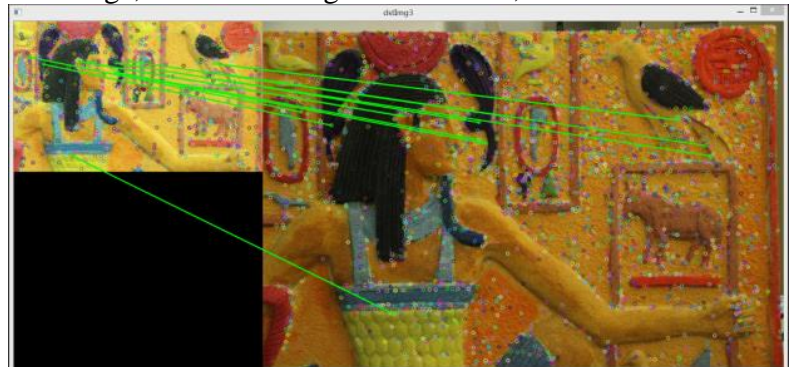

Figure 15. Matching results between local point cloud RGB images and local images

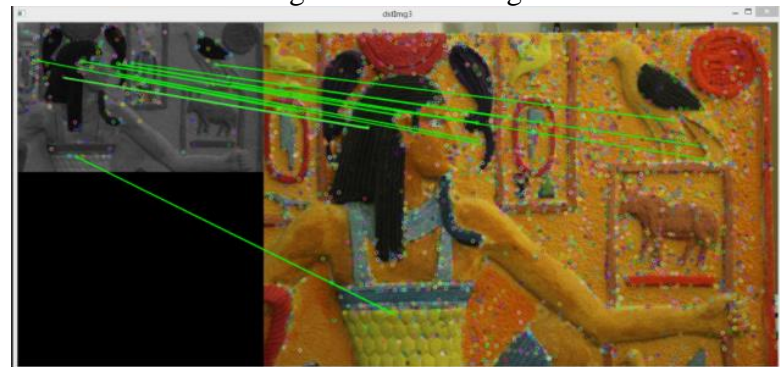

Figure 16. Matching result between local point cloud reflection intensity image and local image

5. Using the same name feature points of optical image and point cloud reflection intensity image, applying the same name feature point and registration model based on Rodrigo matrix, the rough registration of two kinds of data at any angle is realized.In the registration of collinear equation with least square and high precision, the weight reduction of rough handicap is obtained by using the improved standard residual Denmark method, and the result of color point cloud after texture mapping is shown in figure 17;

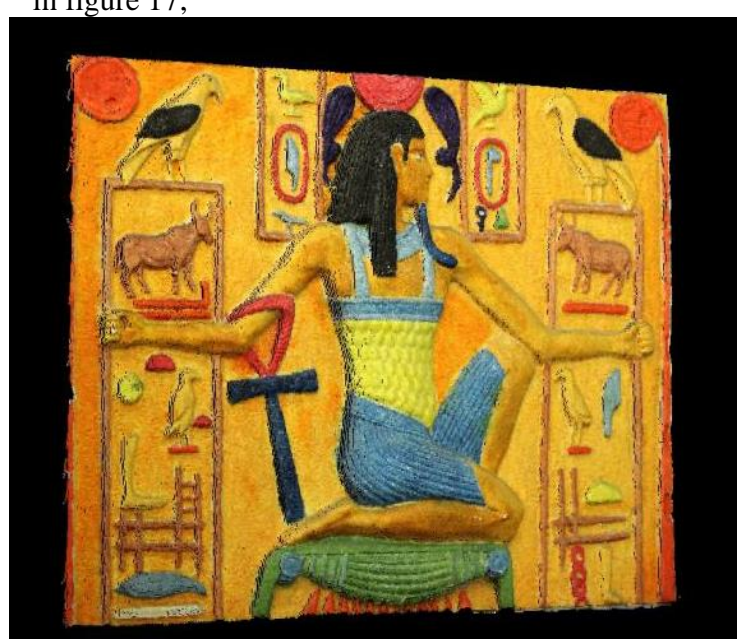

Figure 17. Color point cloud results

It can be seen from figure 17 that the method in this paper is feasible, and the true color point cloud model really reflects the relief properties of sandstone material, and the details are abundant. The registration method from coarse to fine ensures the registration stability and reliability of image and point cloud. The two kinds of non-homologous data are bridged by intermediate data, which to some extent reduces the difference between them, and is more advantageous to the automatic processing of the two kinds of data. But we can also see that there is obvious texture seam phenomenon in the registration result, which is due to the difference of image parameters because there is no normalized processing for local high resolution image.

\section{CONCLUSION}

In this paper, by way of the whole image of the bridge, the affine transformation establishes a link between point cloud reflection intensity image and local image, and find the local point cloud data of local image corresponding to the image by local and local point cloud from coarse to fine registration, automatic high precision registration to realize local high resolution images and point clouds. To a certain extent, the non homologous difference between the optical image and the point cloud image is reduced to a certain extent, which makes a certain promotion for the registration of non homologous data.

\section{REFERENCES}

Geng Zexun, 2010. Digital photogrammetry. Surveying and Mapping Press.

He Peipei, Wan yacun, Yang Wei, Qin Jia Xin, 2015. Automatic registration of laser point clouds and images in urban area based on line characteristics. Acta optica Sinica, 35 (5), pp.352-360. 
Hu Chunmei, Li Tian Shu, 2014. A method of generating lidar reflection intensity image based on center projection. Laser magazine (8), pp. 12-14.

Hu Chunmei,Li Tianshuo,Wang Yan Min, 2015. Research on seamless texture mapping of ground laser radar and close range image data based on deep image. Survey Bulletin (1), pp.66-69.

Li Tianshuo, Wang Yanmin, Hu Chunmei, 2014. Automatic registration of point clouds based on laser reflection intensity. Surveying and mapping Bulletin (S2), pp.143-145.

Qian Jinju, Yang Bisheng, Chen Chi, Dong Zhen, 2015. Automatic registration of ground laser point clouds and texture images. Mapping geographic information, 40 (3), pp.18-20.

Wang Yanmin, Hu Chunmei, 2012. A robust registration method for point cloud and texture images of terrestrial lidar. Journal of Surveying and mapping, 41 (2), pp.266-272.

Zhang Zuxun, Zhang Jianqing, Liao Mingsheng, tension, 1998. remote sensing image high-accuracy automatic registration. Journal of Wuhan University of Surveying and technology, 23 (4), pp.320-323. 